\title{
Performance and Design Investigation of Heavy Lift Tiltrotor with Aerodynamic Interference Effects
}

\author{
Hyeonsoo Yeo \\ Aeroflightdynamics Directorate (AMRDEC) \\ U.S. Army Research, Development, and Engineering Command \\ Ames Research Center, Moffett Field, California
}

\author{
Wayne Johnson \\ Aeromechanics Branch \\ NASA Ames Research Center \\ Moffett Field, California
}

\begin{abstract}
The aerodynamic interference effects on tiltrotor performance in cruise are investigated using comprehensive calculations, to better understand the physics and to quantify the effects on the aircraft design. Performance calculations were conducted for 146,600-1b conventional and quad tiltrotors, which are to cruise at 300 knots at $4000 \mathrm{ft} / 95 \mathrm{deg} \mathrm{F}$ condition. A parametric study was conducted to understand the effects of design parameters on the performance of the aircraft. Aerodynamic interference improves the aircraft lift-to-drag ratio of the baseline conventional tiltrotor. However, interference degrades the aircraft performance of the baseline quad tiltrotor, due mostly to the unfavorable effects from the front wing to the rear wing. A reduction of rotor tip speed increased the aircraft lift-to-drag ratio the most among the design parameters investigated.
\end{abstract}

\section{Notation}

A rotor disk area

$\mathrm{C}_{\mathrm{W}} \quad$ rotor weight coefficient

e Oswald efficiency factor

$\mathrm{L} / \mathrm{D}=\mathrm{WV} / \mathrm{P}$ aircraft effective lift-to-drag ratio

$\mathrm{P} \quad$ aircraft power

q dynamic pressure

$\mathrm{R} \quad$ rotor radius

$\mathrm{S} \quad$ wing area

V flight speed

W gross weight

W/A disk loading

W/S wing loading

$\sigma \quad$ solidity (thrust weighted)

\section{Introduction}

The recent NASA Heavy Lift Rotorcraft Systems Investigation [1] and on-going Joint Heavy Lift (JHL) Concept Design and Analysis (CDA) have renewed the interest in heavy lift aircraft for both civil and military applications. A tiltrotor aircraft configuration has the potential to combine vertical take-off and landing capability with efficient, high-speed cruise flight.

Presented at the American Helicopter Society 63rd Annual Forum, Virginia Beach, VA, May 1-3, 2007. This material is declared a work of the U.S. Government and is not subject to copyright protection in the United States.
Accurate prediction of aircraft performance is essential for the design of future rotorcraft. It is necessary to incorporate rotor/rotor, rotor/wing, and wing/wing interference effects in the performance calculations. The advent of a quad tiltrotor (two wings and four rotors) has increased the importance of aerodynamic interference.

There have been many studies on the aerodynamic interactions between rotor and wing of a conventional tiltrotor in hover due to a significant wing download and its implication on hover performance [2-4]. After Bell Helicopter proposed the development of Quad Tiltrotor (QTR) [5], researchers conducted both experiments [6, 7] and analysis using computational fluid dynamics (CFD) $[8,9]$.

The current study investigates the aerodynamic interference effects on tiltrotor performance in cruise using comprehensive calculations, with the objective to better understand the physics and to quantify the effects on the aircraft design. In this paper, performance calculations were conducted for 146,600-lb conventional and quad tiltrotors, which are to cruise at 300 knots at $4000 \mathrm{ft} / 95 \mathrm{deg} F$ condition. A parametric study was conducted to understand the effects of design parameters on the performance of the aircraft.

\section{Tiltrotor Modeling and Analysis}

The baseline conventional tiltrotor considered is a 20ton payload tiltrotor, which is to cruise at 300 knots 
at $4000 \mathrm{ft} / 95 \mathrm{deg} F$ condition. The configuration of the baseline tiltrotor is shown in Fig. 1. The aircraft has two four-bladed tilting rotors at the wing tips, a high wing, and a horizontal tail. The basic size of the aircraft was determined using the U.S. Army Aeroflightdynamics Directorate's design code RC [11]. Aircraft performance was calculated with the comprehensive rotorcraft analysis CAMRAD II [12], which has demonstrated good performance and airloads correlation with test data [13]. The characteristics of the baseline tiltrotor are summarized in Table 1. The baseline aircraft design parameters are disk loading of W/A $=15$ $\mathrm{lb} / \mathrm{ft}^{2}$, blade loading of $\mathrm{C}_{\mathrm{W}} / \sigma=0.14$, and wing loading of $\mathrm{W} / \mathrm{S}=100 \mathrm{lb} / \mathrm{ft}^{2}$. The airframe and wing parasite drag is $\mathrm{D} / \mathrm{q}=55 \mathrm{ft}^{2}$. This drag value is considered aggressive in terms of rotorcraft trends but achievable from good fixed wing aerodynamic design practice. A hingeless rotor hub was used, with a first blade flap frequency of $1.105 / \mathrm{rev}$. The rotor was modeled as a rigid blade with a flap hinge. Wing and airframe elastic motion was not considered. The rotors rotate with the top blades moving outward in airplane mode.

The baseline quad tiltrotor is developed (not designed by the design code RC) from the baseline conventional tiltrotor, having the same gross weight, disk loading, and airframe size. The configuration of the baseline quad tiltrotor is shown in Fig. 2. The characteristics of the baseline quad tiltrotor are summarized in Table 2. The rotor size was determined to maintain same disk loading as the baseline conventional tiltrotor. The front wing span followed from maintaining the same clearance between the two rotors, and the front wing chord was determined by maintaining the same aspect ratio as the baseline conventional tiltrotor wing. The rear wing span was chosen as $40 \%$ larger than the front wing span. The rear wing chord was chosen to have the same chord $(15.21 \mathrm{ft})$ as the front wing from the tips to the middle of the semi-span and then linearly increased to $17.35 \mathrm{ft}$ at the center line. The quarter chord line of the rear wing was kept straight. This design approach resulted in wing loading of $\mathrm{W} / \mathrm{S}=67.16 \mathrm{lb} / \mathrm{ft}^{2}$. The rear rotors and wing are located $5.02 \mathrm{ft}$ above the front rotors and wing. The blade structural properties were scaled to have the same first blade flap frequency as the conventional tiltrotor $(1.105 / \mathrm{rev})$. The rotors rotate with the top blades moving outward in airplane mode.

Performance calculations were conduced at the design cruise of 300 knots at $4000 \mathrm{ft} / 95 \mathrm{deg} \mathrm{F}$ condition. Rotor/rotor, rotor/wing, and wing/wing interferences were accounted for using the vortex wake model. The current analysis does not include fuselage model, which is known to be important for oscillatory interference of wing on rotor, but not usually necessary for wing mean induced drag. No nacelle model was considered, thus any end plating effect was neglected. Typical wake geometries and blade and wing lift distributions for the baseline conventional and quad tiltrotor are shown in Figs. 3 and 4, respectively. Only the tip vortices, which dominate the interference, are drawn in these figures, but there was a full vortex lattice behind each blade and wing. Wing wake model consists of vortex lattice in the near wake behind the wing with 32 aerodynamic panels, rolling up to tip vortices (with shed wake panels between) in the far wake that interferes with the rotors and other wing. Thus, comparable models used for both wing and rotor wakes in this investigation of the interference. A constant vortex core radius of $20 \%$ chord $(0.2 \mathrm{c})$ was used for both conventional and quad tiltrotor. The effects of vortex core size on the aircraft performance was discussed in the later section.

For the conventional tiltrotor, the aircraft was trimmed using longitudinal stick (connected to the elevator), governor, and pitch attitude to obtain longitudinal and vertical force and pitching moment equilibrium of the aircraft. For some cases, rotor flapping was also trimmed to zero using rotor cyclic pitch to reduce loads; thus there were 3 or 7 trim variables for cruise.

For the quad tiltrotor, the aircraft was trimmed using governor and front and rear wing pitch angles. The governor was used to achieve longitudinal force equilibrium and the front and rear wing pitch angles were used for each wing to carry half of the gross weight. Rotor flapping was also trimmed to zero using rotor cyclic pitch; thus there were 11 trim variables for cruise.

\section{Performance and Design Analysis}

\section{Conventional Tiltrotor}

Performance results for a conventional tiltrotor at the design cruise of 300 knots at $4000 \mathrm{ft} / 95 \mathrm{deg} F$ condition are shown here. The performance was calculated using nonuniform inflow with prescribed wake geometry. Figures 5 through 7 show the interference effects on the wing for the baseline configuration. The interference velocity from the rotors on the wing is shown in Fig. 5. The interference varies with rotor azimuth and exhibits $4 /$ rev variation due to the 4 -bladed rotors. The maximum interference was observed at 30 degree azimuth angle, when the blade tip vortex of the rotor passes the wing quarter chord line. These interference velocities reduce total induced velocity along the wing span as shown in Fig. 6. Without interference, the induced velocity distribution is same as that of a fixed wing. The interference has a beneficial effect on the wing performance, reducing wing induced power as shown in 
Fig. 7. The interference effect did not change wing profile power. This observation, the reduction of wing drag due to favorable rotor swirl provided by up-inboard rotating rotors, was also reported in Refs. [10,14].

A parametric study was conducted to understand the effects of design parameters on the performance of the aircraft. Table 3 shows the design parameters investigated and the rotor and wing geometry variations are illustrated in Fig. 8. First case was the change of rotor rotational direction. Second case was the increase of disk loading to $16.6 \mathrm{lb} / \mathrm{ft}^{2}$ (reduction of rotor blade radius by $5 \%$ ). To maintain the same blade loading, blade chord was increased accordingly. Third case was reduction of cruise tip speed, to increase the propulsive efficiency of the rotor. Fourth case was the reduction of wing angle of attack relative to the fuselage (thus relative to the rotors), to investigate the effect of lift sharing between rotor and wing. Fifth case was the increase of wing span. To maintain the same wing loading, wing chord was reduced accordingly. In this case, the rotors stay at the same wing span as the baseline. Sixth case was the increase of wing span, same as the fifth case, but the rotors move to the wing tips. Seventh case was the increase of wing span, rotors at the wing tips, and increase of rotor blade radius (decrease disk loading to $11.9 \mathrm{lb} / \mathrm{ft}^{2}$ ). To maintain the same blade loading, blade chord was decreased accordingly.

Figure 9 shows the performance results in terms of aircraft lift-to-drag ratio $\mathrm{L} / \mathrm{D}=\mathrm{WV} / \mathrm{P}$, calculated without accessory or other losses, all for the design cruise condition of 300 knots. Rotor/rotor and rotor/wing interferences were accounted for using a vortex wake model for both the rotor and the wing, and the performance results with interference effects are compared with those without interference effects. Two trim strategies were used: with flapping trim and without flapping trim. The interference effects changed the aircraft lift-to-drag ratio by up to $2.1 \%$ for the parametric variations investigated. The reduction of rotor tip speed (C3) increased the aircraft lift-to-drag ratio the most and the increase of wing span (C5) also has a beneficial effect. The change of rotor rotational direction (C1) decreased the aircraft lift-to-drag ratio significantly and this effect can only be observed with interference included in the calculation. The total effect of changing the rotor direction of rotation was $-3.0 \%$ of L/D. Rotor disk loading change ( $\mathrm{C} 2$, and $\mathrm{C} 7$ compared to $\mathrm{C} 6$ ) has a small influence on the aircraft cruise performance. In general, flapping trim reduces the aircraft lift-to-drag ratio by up to $1.4 \%$.

Figures 10 and 11 show the rotor propulsive efficiency and wing drag (induced + parasite), respectively. These are the same calculations as in Fig. 9, except that individual performance components are compared. For the baseline case, there is a significant reduction of the wing induced drag because of the favorable combination of the rotor wake and the wing; and a slight increase in rotor propulsive efficiency because of the nonuniform flow field from the wing interference. The change of rotor rotational direction increases rotor propulsive efficiency somewhat. However, it also increases the wing induced drag significantly, thus overall performance penalty was observed. The reduction of rotor tip speed (C3) increased the rotor propulsive efficiency as well as decreased the wing drag. Thus, the most performance improvement was obtained. The tip speed value of $350 \mathrm{ft} / \mathrm{sec}$ was selected based on the optimum aircraft performance as shown in Fig. 13. The rotor tip speed was varied from 250 to $450 \mathrm{ft} / \mathrm{sec}$ and the optimum cruise performance was found at $350 \mathrm{ft} / \mathrm{sec}$ tip speed. Further reductions in rotor rotational speed did not improve the aircraft L/D. The reduction of wing angle of attack (C4) changed lift sharing between the rotor and wing; reduced wing lift by about $4000 \mathrm{lb}$ and increased rotor lift by about 4000 $\mathrm{lb}$. The reduced wing lift decreased wing induced drag and the increased rotor lift increased rotor induced drag. However, the reduced wing angle of attack also changed wing tip vortex trajectories in a way to increase beneficial interference effects, thus the rotor propulsive efficiency was not changed much. The net effect of the reduction of wing angle of attack was a performance improvement. The increase of wing span (C5, and decrease of wing chord) decreased the wing drag (mostly induced drag), but slightly decreased the rotor propulsive efficiency. When the rotor moved to the wing tip (C6) for the increased wing span (C5), wing drag was reduced due to increased beneficial interference effects.

Figure 12 shows the Oswald efficiency factor for the wing. Planar wings with the Oswald efficiency equal to 1.0 have an elliptical lift distribution and will produce the minimum induced drag for a given wing section and span. Ideal induced drag (elliptical loading on entire wing system) is

$$
\mathrm{D}_{\text {ideal }} / \mathrm{q}=(\mathrm{L} / \mathrm{q})^{2} /\left(\pi * \operatorname{span}^{2}\right)
$$

Thus, Oswald efficiency factor is

$$
\begin{aligned}
\mathrm{e} & =\left(\mathrm{D}_{\text {ideal }} / \mathrm{q}\right) /\left(\mathrm{D}_{\text {induced }} / \mathrm{q}\right) \\
& =(\mathrm{L} / \mathrm{q})^{2} /\left(\pi * \operatorname{span}^{2} *\left(\mathrm{D}_{\text {induced }} / \mathrm{q}\right)\right)
\end{aligned}
$$

The Oswald efficiency factor for the baseline case is 0.976 without interference and 1.078 with interference. Those values are very high for a rectangular wing and 
the 32 aerodynamic panels used in the wing aerodynamic calculation appear not enough to accurately capture sudden lift drop near the wing tip (aerodynamic panel size near the wing tip is $2 \%$ of the wing span). However, the 32 aerodynamic panels for the wing are considered enough to investigate the interference effects. Without interference, the Oswald efficiency factor change was negligible. The wing drag reduction observed for the cases 5,6 , and 7 was compensated by the wing span increase, thus the net wing efficiency was unchanged. The wing efficiency due to interference effects are consistent with the wing drag results shown in Fig. 11. The interference has a beneficial effect on the wing efficiency for most of the cases investigated, reducing wing induced drag.

\section{Quad Tiltrotor}

Performance results for a quad tiltrotor configuration are shown here. Figures 14 through 16 show the interference effects on the wing at the design cruise condition of 300 knots. The interference velocity from the rotors on the wing is shown in Fig. 14. The interference varies with rotor azimuth and exhibits 4/rev variation due to the 4bladed rotors. The interference on the front wing is similar to that of a conventional tiltrotor. The maximum interference on the front wing was observed at 30 degree azimuth angle, when the blade tip vortex of the rotor passes the wing quarter chord line. The interference velocity values on the front wing are mostly positive due to positive interference velocities from the rear wing. The interference on the rear wing is very complicated because several sources affect it. The most dominant influence comes from the front wing, determines Wshape distribution. The two humps at \pm 0.25 originate from the two rear rotors. These interference velocities reduce total induced velocity along the front wing span and significantly increase total induced velocity along the rear wing span as shown in Fig. 15. Without interference, the induced velocity distribution of the rear rotor is somewhat different from the front wing because of the increased chord near mid-span. The interference has a beneficial effect on the front wing performance, but degrades the rear wing performance as shown in Fig. 16. The interference effect did not change wing profile power.

Figure 17 quantifies the interference effect on the aircraft cruise performance, for the baseline configuration. The required power changes due to interference are shown. The interference effects between the front rotors and the front wing and between the rear rotors and the rear wing reduce required power. The front wing has a beneficial influence on the rear rotor power. The rear wing also has a beneficial influence on the front wing (positive interference velocity reduced total induced velocity, and thus reduced wing induced power). The front rotors increase both rear rotor power and rear wing power, although the effect is not significant. The most dominant effect is from the front wing to the rear wing. It increases the required power of the rear wing by $810 \mathrm{HP}$ (negative interference velocity increased total induced velocity, and thus increased wing induced power at most of the wing span), thus increases the aircraft total required power.

As the vortex core size grows with time (wake age) due to viscous diffusion, the effects of vortex core size on the aircraft performance was investigated. It should be noted that the constant vortex core radius of $20 \%$ chord $(0.2 \mathrm{c})$ was used for all the calculations shown in this paper. Without knowing an accurate core growth rate, a simple way to examine the effect of core growth is to use larger core size for both rotors and wings when the interference velocity is calculated. Figure 18 shows the effects of vortex core size on the rotor propulsive efficiency and wing drag. The calculations were made with two vortex core sizes $(0.5 \% \mathrm{c}$ and $1.0 \% \mathrm{c})$ and the results are compared with the baseline values. As the vortex core size increases, the front rotor propulsive efficiency increases and the rear wing drag decreases. However, the rear rotor propulsive efficiency decreases and the front wing drag slightly increases at the same time. Thus, the total effect of changing the vortex core size on the aircraft performance is negligible.

A parametric study was conducted to understand the effects of design parameters on the performance of the aircraft. Table 4 shows the design parameters investigated and the rear rotor and wing geometry variations are illustrated in Fig. 19. First case was the change of rotor rotational direction. Second case was reduction of cruise tip speed for all four rotors, to increase the propulsive efficiency of the rotor. Third case was reduction of both front and rear wing chords to obtain wing loading of $\mathrm{W} / \mathrm{S}=100 \mathrm{lb} / \mathrm{ft}^{2}$, which is the value for the conventional tiltrotor. In this case, wing span was maintained same as the baseline value. Fourth case was rear rotors moved to inboard to make them directly behind the front rotors. Fifth case was rear rotors moved to inboard same as the fourth case, but the rear wing span decreased to match front wing span (102.6 ft to $73.3 \mathrm{ft}$ ). To maintain the same wing loading, wing chord was increased accordingly. Sixth case was increase of rear wing span by $20 \%$ (102.6 $\mathrm{ft}$ to $123.1 \mathrm{ft}$ ) and rear rotors moved to the wing tip. To maintain the same wing loading, wing chord was decreased accordingly. Seventh case was rear rotors and wing moved down to the same height as the front rotors and wings (baseline separation $5.02 \mathrm{ft}$ ).

Figure 20 shows the performance results in terms of 
aircraft lift-to-drag ratio $\mathrm{L} / \mathrm{D}=\mathrm{WV} / \mathrm{P}$, calculated without accessory or other losses, all for the design cruise condition of 300 knots. The performance was calculated using nonuniform inflow with prescribed wake geometry. Rotor/rotor, rotor/wing, and wing/wing interference was accounted for using a vortex wake model for both the rotor and the wing, and the performance results with interference effects are compared with those without interference effects. Zero flapping trim was used for all the results for the quad tiltrotor. The interference effects changed the aircraft lift-to-drag ratio by up to $7.8 \%$ for the parametric variations investigated. The reduction of rotor tip speed (C2) and increase of wing loading (C3) increased the aircraft lift-to-drag ratio the most and the increase of rear wing span (C6 compared to C5) also has a beneficial effect. The change of rotor rotational direction $(\mathrm{C} 1)$ and the move of the rear rotors and wings to the same height as the front rotors and wing (C7) decreased the aircraft lift-to-drag ratio significantly and these effects can only be observed with interference included in the calculation. The move of the front rotors inboard (C4) has negligible influence on the aircraft liftto-drag ratio, however the reduction of rear wing span (C5) significantly reduced the aircraft lift-to-drag ratio.

Figures 21 and 22 show the rotor propulsive efficiency and wing drag, respectively. These are the same calculations as in Fig. 20, except that individual performance components are compared. For the baseline case, there is a reduction of the front wing induced drag and a significant increase of the rear wing induced drag because of the nonuniform flow field from the rotor and wing interference; and a slight reduction in the front rotor propulsive efficiency and an increase of rear rotor propulsive efficiency because of the combination of the interference and the front rotor thrust decrease and rear rotor thrust increase. The change of rotor rotational direction $(\mathrm{C} 1)$ increases the front rotor propulsive efficiency. However, it also increases the wing drag significantly, thus overall performance penalty was observed. The reduction of rotor tip speed (C2) increased the rotor propulsive efficiency as well as decreased the wing drag. Thus, the most performance improvement was obtained. The increase of wing span (C6 compared to C5) decreased the wing drag, but slightly decreased the rotor propulsive efficiency.

Without interference effect, the front wing drag values do not change with the parametric variations except for the wing loading change (C3). With interference effect, the change of rotor rotational direction $(\mathrm{C} 1)$ increases the front wing drag significantly, as for the conventional tiltrotor. The increase of wing loading $(\mathrm{C} 3$, wing chord was reduced with same span as baseline) significantly reduced the front wing drag. The significant drag reduction came from profile drag reduction due to the reduced chord. However, induced drag slightly increased.

The rear wing drag values show significant variations with the parametric variations and the rear wing drag values always increased with the interference effects for the parametric variations investigated. The biggest penalty came from the reduction of the rear wing span (C5) due to the increased induced and interference drag. The biggest benefit came from the increase of wing loading (C3) and the increase of the rear wing span (C6 compared to C5). However, the reasons for the wing performance improvement are different. The increase of wing loading (C3) resulted in the reduction of profile drag, but the the increase of the rear wing span (C5) resulted in the reduction of induced and interference drag.

Figure 23 shows the Oswald efficiency factor for the entire wing. Although each wing has its own efficiency factor, comparison of the entire wing efficiency was considered more valuable for the design parametric study. Thus, the Oswald efficiency factor definition used in the plot is same as Eqn. (2). However, total wing lift ( $\mathrm{L}_{\text {total }}$ $=\mathrm{L}_{\text {front }}+\mathrm{L}_{\text {rear}}$ ), and total wing induced drag values were used rather than individual wing lift and drag values, but the longer wing span, which is rear wing span for the parametric variations investigated here, was used. With this definition of e, the ideal efficiency is $\mathrm{e}=1.0$ if the tandem wings are in the same plane, but greater than 1.0 with vertical separation of the wings. The cases without interference exclude the wing/wing interference as well as the wing/rotor and rotor/wing interference; using this definition of efficiency, two independent wings each with elliptical loading would give

$$
\mathrm{e}=4 /\left(1+\left(\operatorname{span}_{\text {rear }} / \operatorname{span}_{\text {front }}\right)^{2}\right)
$$

Without interference, the Oswald efficiency factor change was negligible except for $\mathrm{C} 5$ and $\mathrm{C} 6$, where rear wing span decreased and increased, respectively. Although a significant wing drag change was observed for the $\mathrm{C} 3$, the Oswald efficiency factor change is negligible because the wing drag change came from profile drag reduction. The Oswald efficiency factor is almost 2.0 for the C5, which is similar to a bi-plane with two wings separated. The interference has an unfavorable effect on the wing efficiency for all the cases investigated.

\section{Conclusions}

A performance and design investigation was conduced for 146,600-lb conventional and quad tiltrotors, which are to cruise at 300 knots at $4000 \mathrm{ft} / 95 \mathrm{deg} \mathrm{F}$ condition. The aerodynamic interference effects were included in 
the comprehensive calculations to better understand the physics and to quantify the effects on the aircraft design.

From this study the following conclusions were obtained:

\section{Conventional tiltrotor}

1. Interference effects improves the aircraft lift-todrag ratio of the baseline conventional tiltrotor. The interference velocities reduce total induced velocity along the wing span, and thus reduce wing induced power. The interference effect has very small influence on wing profile power and rotor propulsive efficiency.

2. The reduction of rotor tip speed increased the aircraft lift-to-drag ratio the most among the design parameters investigated and the increase of wing span also has a beneficial effect on the aircraft performance.

3. The change of rotor rotational direction decreased the aircraft lift-to-drag ratio significantly and this effect can only be observed with interference included in the calculation.

\section{Quad tiltrotor}

1. Interference effects degrade the aircraft performance of the baseline quad tiltrotor. The beneficial interferences occur between the front rotors and the front wing and between the rear rotors and the rear wing and from the front wing to the rear rotors and the rear wing to front wing. The unfavorable interferences occur from front rotors to the rear rotors and rear wing, from the front wing to the rear wing. The most dominant effect is from the front wing to the rear wing and it increases the rear wing total induced power significantly and thus decrease the aircraft lift-to-drag ratio.

2. The reduction of rotor tip speed and increase of wing loading increased the aircraft lift-to-drag ratio the most among the design parameters investigated and the increase of rear wing span also has a favorable effect on the aircraft performance.

3. The change of rotor rotational direction decreased the aircraft lift-to-drag ratio significantly and this effect can only be observed with interference included in the calculation.

\section{References}

[1] Johnson, W., Yamauchi, G. K., and Watts, M. E., "Design and Technology Requirements for Civil Heavy Lift Rotorcraft," Proceedings of the American Helicopter Society Vertical Lift Aircraft Design Conference, San Francisco, CA, January 2006.

[2] Felker, F. F., Maisel, M. D., and Betzina, M. D., "Full-scale Tilt-Rotor Hover Performance," Journal of the American Helicopter Society, Vol. 31, No. 2, April 1986.

[3] Felker, F. F., and Light, J. S, "Aerodynamic Interactions Between a Rotor and Wing in Hover," Journal of the American Helicopter Society, Vol. 33, No. 2, April 1988.

[4] Wood, T. L., and Peryea, M. A., "Reduction of Tiltrotor Download," Journal of the American Helicopter Society, Vol. 40, No. 3, July 1995.

[5] Snyder, D., "The Quad Tiltrotor: Its Beginning and Evolution," Proceedings of the American Helicopter Society 56th Annual Forum, Virginia Beach, VA, 2000.

[6] Radhakrishnan, A., and Schmitz, F. H., "An Experimental Investigation of a Quad Tilt Rotor In Ground Effect," Proceedings of the 21st Applied Aerodynamics Conference, Orlando, FL, June 2003.

[7] Radhakrishnan, A., and Schmitz, F. H., "Quad Tilt Rotor Download and Power Measurements in Ground Effect," Proceedings of the 24th Applied Aerodynamics Conference, San Francisco, CA, June 2006.

[8] Gupta, V., and Baeder, J. D., "Quad Tiltrotor Aerodynamics in Ground Effect," Proceedings of the American Helicopter Society 58th Annual Forum, Montreal, Canada, June 2002.

[9] Gupta, V., and Baeder, J. D., "Investigation of Quad Tiltrotor Aerodynamics in Forward Flight Using CFD," Proceedings of the 20th Applied Aerodynamics Conference, St. Louis, MO, June 2002.

[10] McVeigh, M. A., Grauer, W. K., and Paisley, D. J., "Rotor/Airframe Interactions on Tiltrotor Aircraft," Journal of the American Helicopter Society, Vol. 35, No. 3, July 1990.

[11] Preston, J., and Peyran, R., "Linking a SolidModeling Capability with a Conceptual Rotorcraft Sizing Code," Proceedings of the American Helicopter Society Vertical Lift Aircraft Design Conference, San Francisco, CA, January 2000. 
[12] Johnson, W., "Technology Drivers in the Development of CAMRAD II," Proceedings of the American Helicopter Society Aeromechanics Specialist Meeting, San Francisco, CA, January 1994.

[13] Johnson, W., "Calculation of Tilt Rotor Aeroacoustic Model (TRAM DNW) Performance, Airloads, and Structural Loads," American Helicopter Society Aeromechanics Specialists' Meeting, Atlanta, GA, November 2000.

[14] Drees, J. M., "Prepare for the 21st Century - The 1987 Alexander A. Nikolsky Lecture," Journal of the American Helicopter Society, Vol. 32, No. 3, July 1987. 
Table 1: Characteristics of baseline tiltrotor design

\begin{tabular}{ll}
\hline \hline mission GW $(\mathrm{lb})$ & 146600 \\
cruise speed $(\mathrm{kt})$ & 300 \\
rotor diameter $(\mathrm{ft})$ & 78.88 \\
disk loading W/A $\left(\mathrm{lb} / \mathrm{ft}^{2}\right)$ & 15 \\
$C_{W} / \sigma($ geometric) & 0.140 \\
$C_{W} / \sigma($ thrust weighted) & 0.154 \\
tip speed $(\mathrm{ft} / \mathrm{sec})$ & $750 / 626$ \\
solidity $(\mathrm{geometric})$ & 0.0989 \\
number blades & 4 \\
blade chord $(75 \% \mathrm{R}, \mathrm{ft})$ & 2.79 \\
blade taper ratio & 0.7 \\
aircraft drag $\mathrm{D} / \mathrm{q}\left(\mathrm{ft}^{2}\right)$ & 55.0 \\
wing loading $\left(\mathrm{lb} / \mathrm{ft}^{2}\right)$ & 100 \\
wing area $\left(\mathrm{ft}^{2}\right)$ & 1466 \\
wing span $(\mathrm{ft})$ & 96.4 \\
\hline \hline
\end{tabular}

Table 2: Characteristics of baseline quad tiltrotor design

\begin{tabular}{ll}
\hline \hline mission GW $(\mathrm{lb})$ & 146600 \\
cruise speed $(\mathrm{kt})$ & 300 \\
rotor diameter $(\mathrm{ft})$ & 55.78 \\
disk loading W/A $\left(\mathrm{lb} / \mathrm{ft}^{2}\right)$ & 15 \\
$C_{W} / \sigma($ geometric) & 0.140 \\
$C_{W} / \sigma($ thrust weighted) & 0.154 \\
tip speed $(\mathrm{ft} / \mathrm{sec})$ & $750 / 626$ \\
solidity (geometric) & 0.0989 \\
number blades & 4 \\
blade chord $(75 \% \mathrm{R}, \mathrm{ft})$ & 1.97 \\
blade taper ratio & 0.7 \\
aircraft drag $\mathrm{D} / \mathrm{q}\left(\mathrm{ft}^{2}\right)$ & 60.3 \\
wing loading $\left(\mathrm{lb} / \mathrm{ft}^{2}\right)$ & 67.2 \\
front wing area $\left(\mathrm{ft}^{2}\right)$ & 848 \\
rear wing area $\left(\mathrm{ft}^{2}\right)$ & 1335 \\
front wing span $(\mathrm{ft})$ & 73.3 \\
rear wing span $(\mathrm{ft})$ & 102.6 \\
\hline \hline
\end{tabular}


Table 3: Parametric variations of conventional tiltrotor

\begin{tabular}{ll}
\hline \hline Case 1 (C1) & change of rotor rotational direction \\
Case 2 (C2) & increase of disk loading (reduction of rotor blade radius by 5\%) \\
Case 3 (C3) & reduction of cruise rotor tip speed to $350 \mathrm{ft} / \mathrm{sec}$ \\
Case 4 (C4) & reduction of wing angle of attack by $3 \mathrm{deg}$ \\
Case 5 (C5) & increase of wing span by 10\% \\
Case 6 (C6) & increase of wing span by 10\% and rotors move to the wing tip \\
Case 7 (C7) & increase of wing span by 10\% and rotors move to the wing tip and increase of rotor blade radius by $12.2 \%$ \\
\hline \hline
\end{tabular}

Table 4: Parametric variations of quad tiltrotor

\begin{tabular}{ll}
\hline \hline Case 1 (C1) & change of rotor rotational direction \\
Case 2 (C2) & reduction of cruise rotor tip speed to $350 \mathrm{ft} / \mathrm{sec}$ \\
Case 3 (C3) & reduction of wing chord to obtain wing loading of $100 \mathrm{lb} / \mathrm{ft}^{2}$ \\
Case 4 (C4) & rear rotors moved to inboard to make them directly behind front rotors \\
Case 5 (C5) & rear rotors moved to inboard and rear wing span decreased to match front wing span \\
Case 6 (C6) & increase of rear wing span by 20\% and rear rotors moved to the wing tip \\
Case 7 (C7) & rear rotors and wing moved down to the same height as front rotors and wings \\
\hline \hline
\end{tabular}




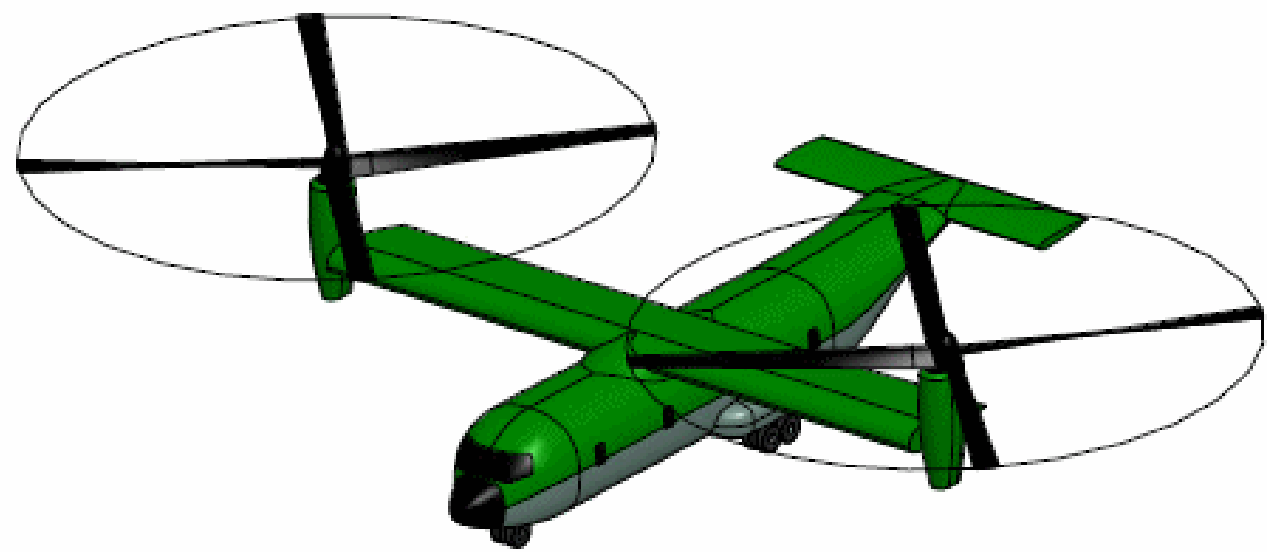

Fig. 1: Baseline tiltrotor configuration (courtesy Gerardo Nunez of U.S. Army, AFDD)

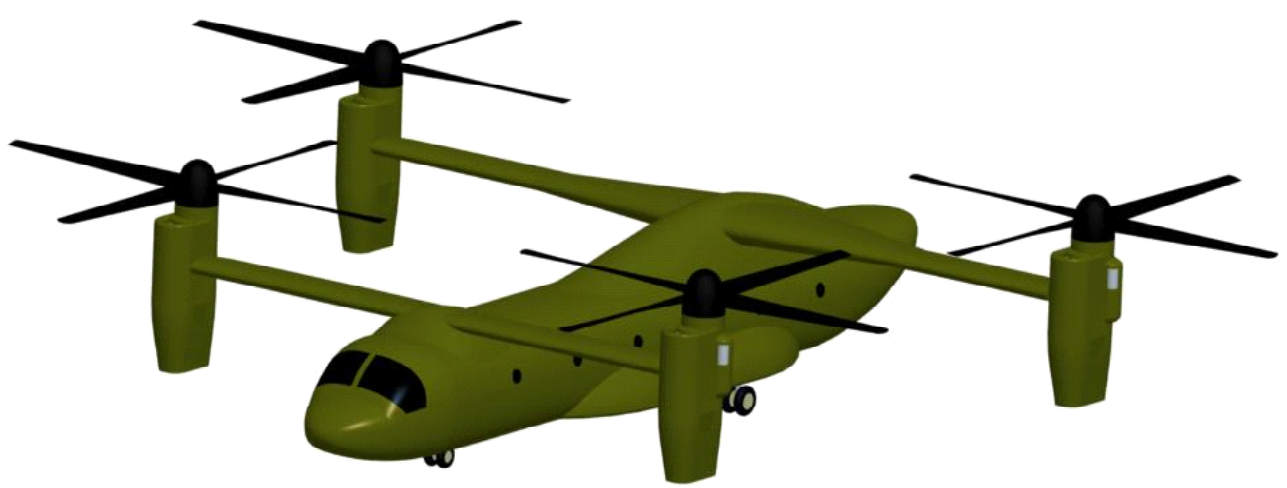

Fig. 2: Baseline quad tiltrotor configuration (courtesy Gerardo Nunez of U.S. Army, AFDD) 


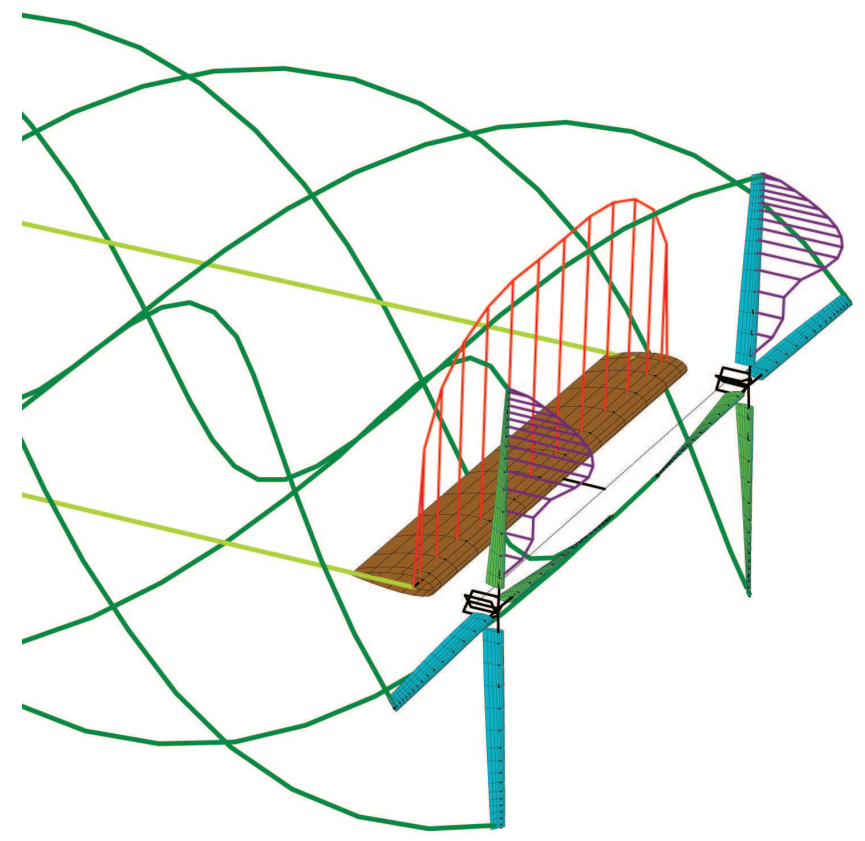

Fig. 3: Wake geometry of conventional tiltrotor

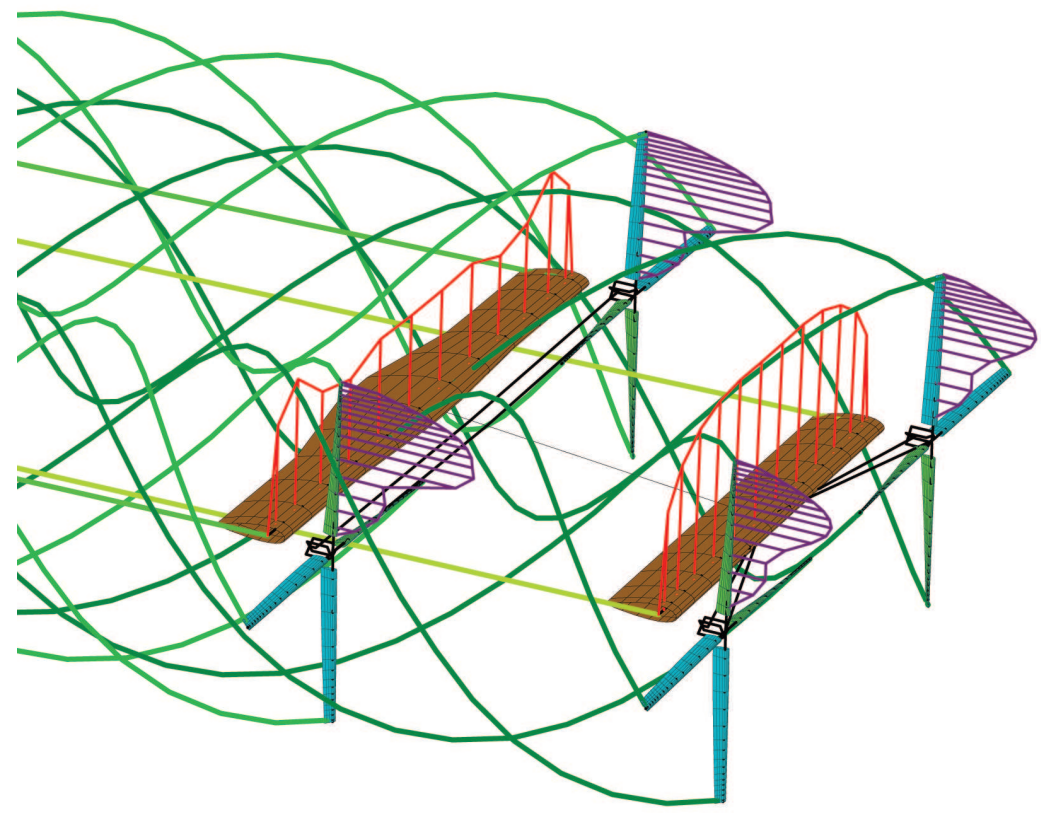

Fig. 4: Wake geometry of quad tiltrotor 


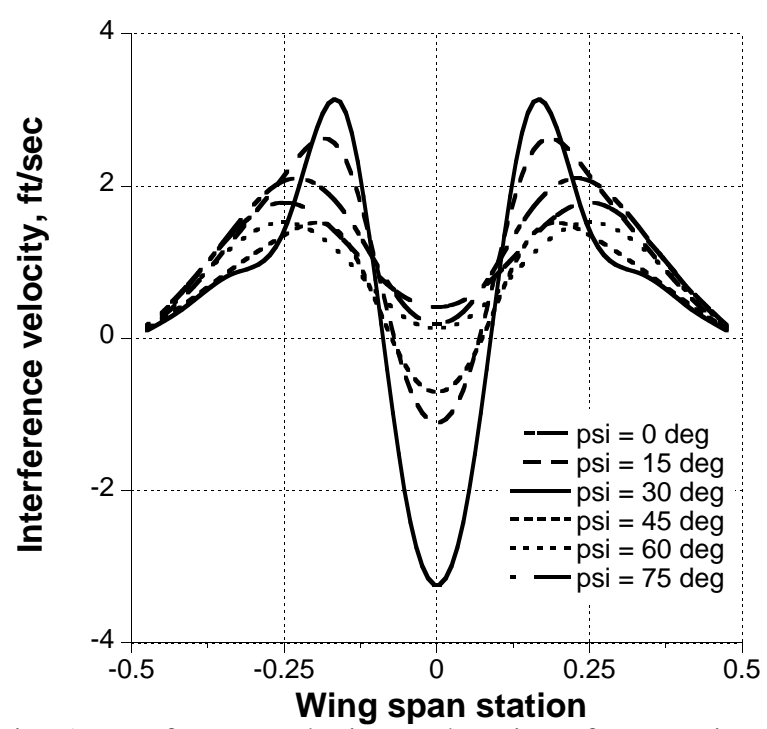

Fig. 5: Interference velocity on the wing of conventional tiltrotor (positive upward)

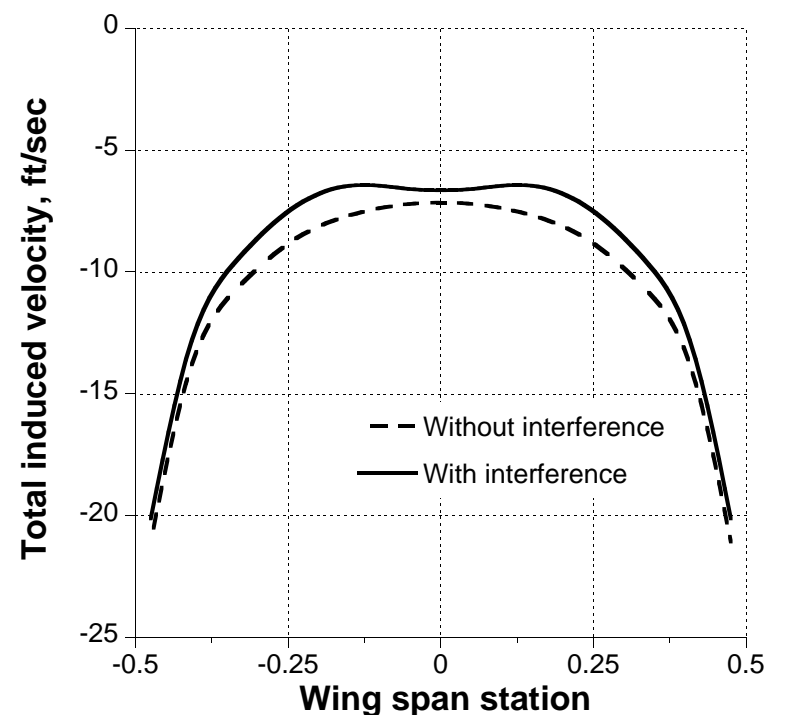

Fig. 6: Total wing induced velocity of conventional tiltrotor (positive upward)

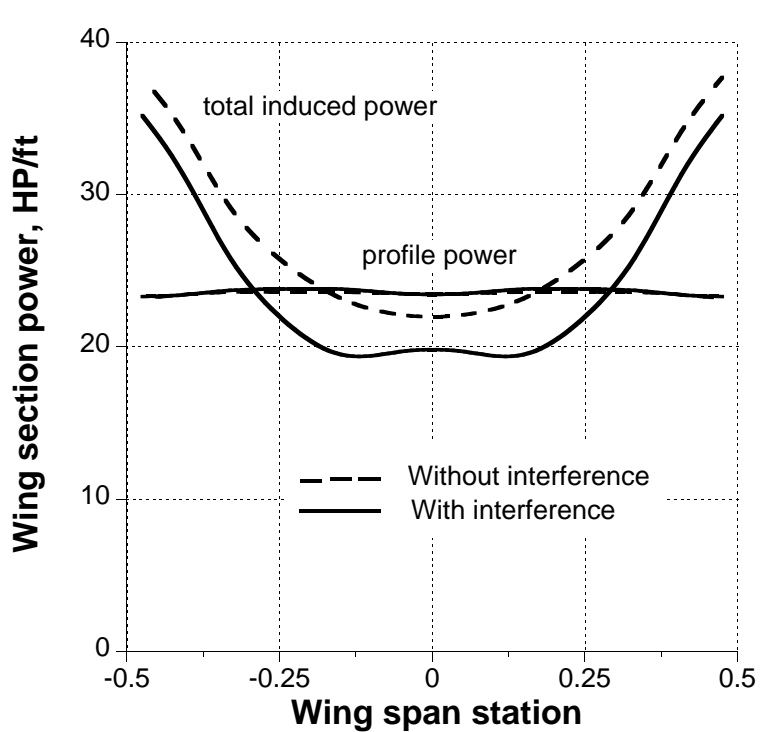

Fig. 7: Wing section power of conventional tiltrotor

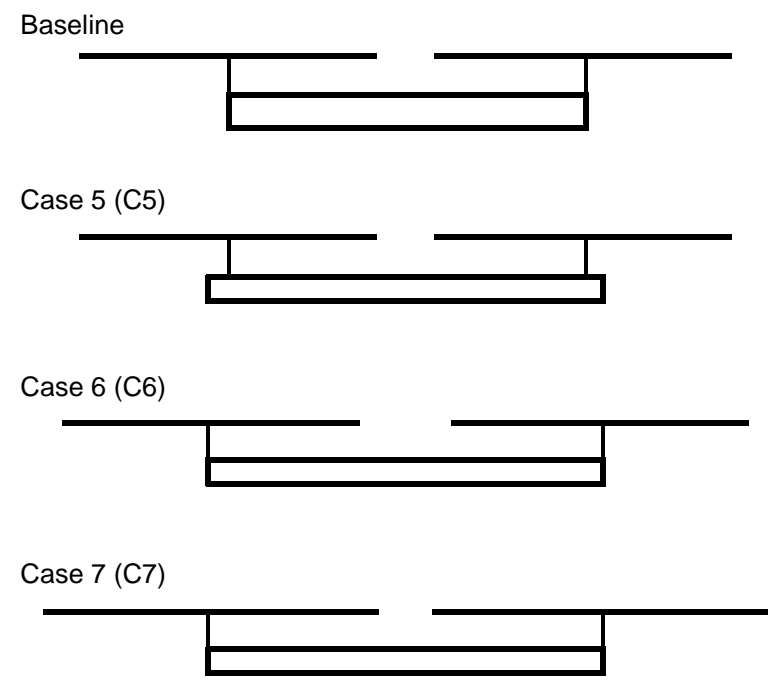

Fig. 8: Rotor and wing geometry variations for conventional tiltrotor 


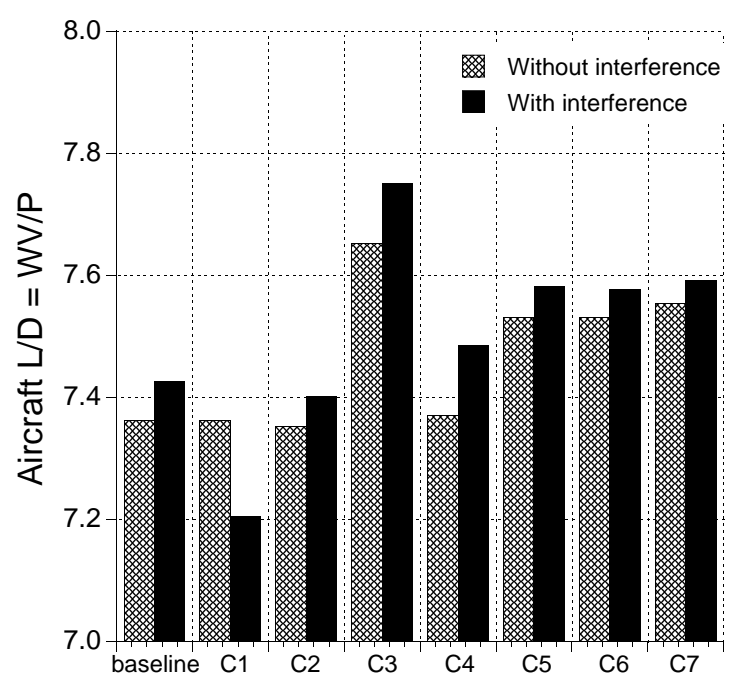

(a) Flapping trim

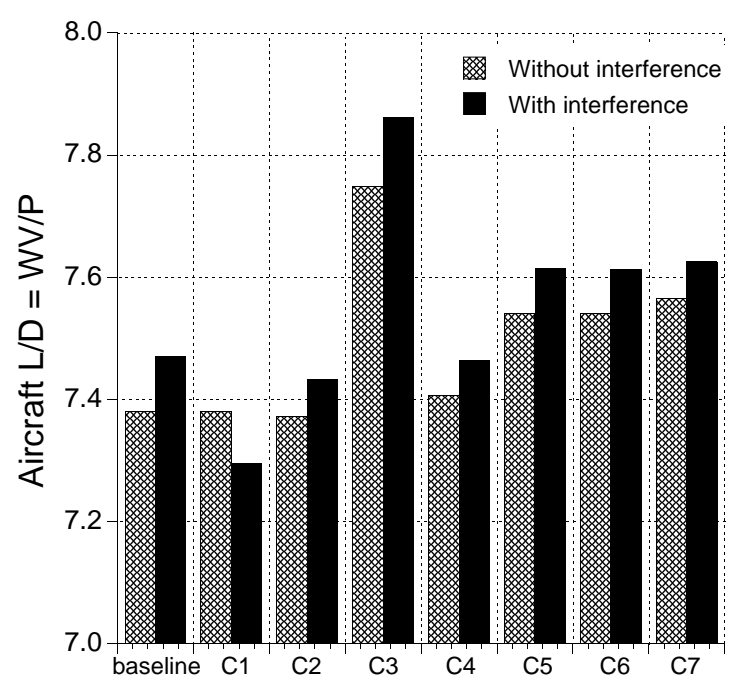

(b) Without flapping trim

Fig. 9: Aircraft lift-to-drag ratio of conventional tiltrotor

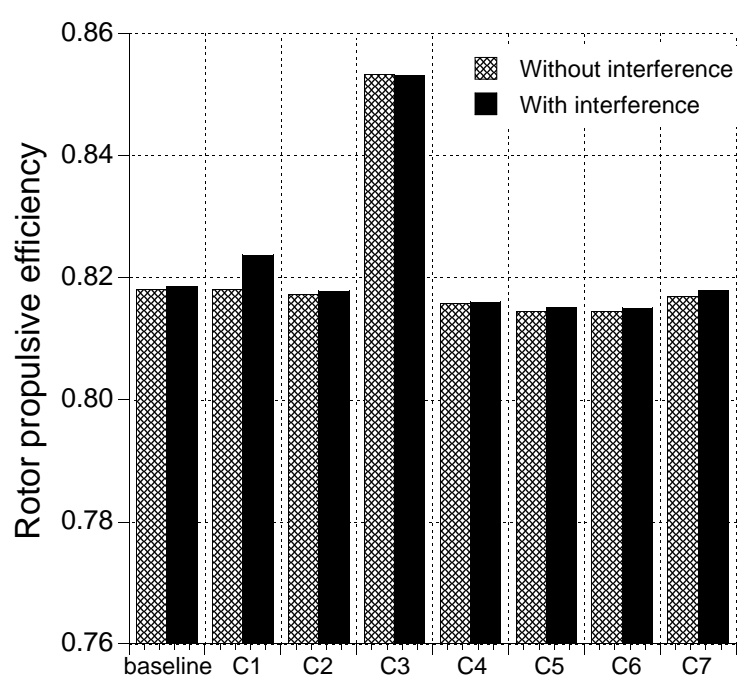

(a) Flapping trim

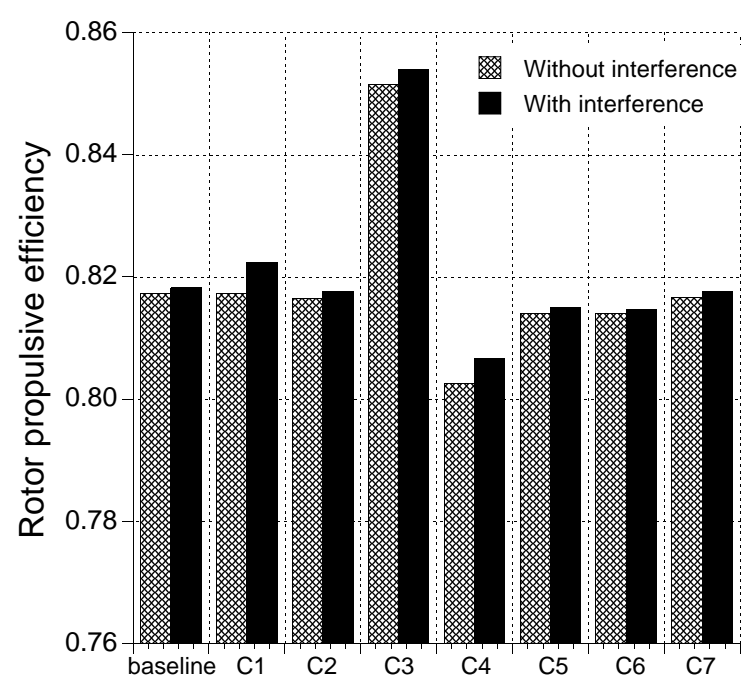

(b) Without flapping trim

Fig. 10: Rotor propulsive efficiency of conventional tiltrotor 


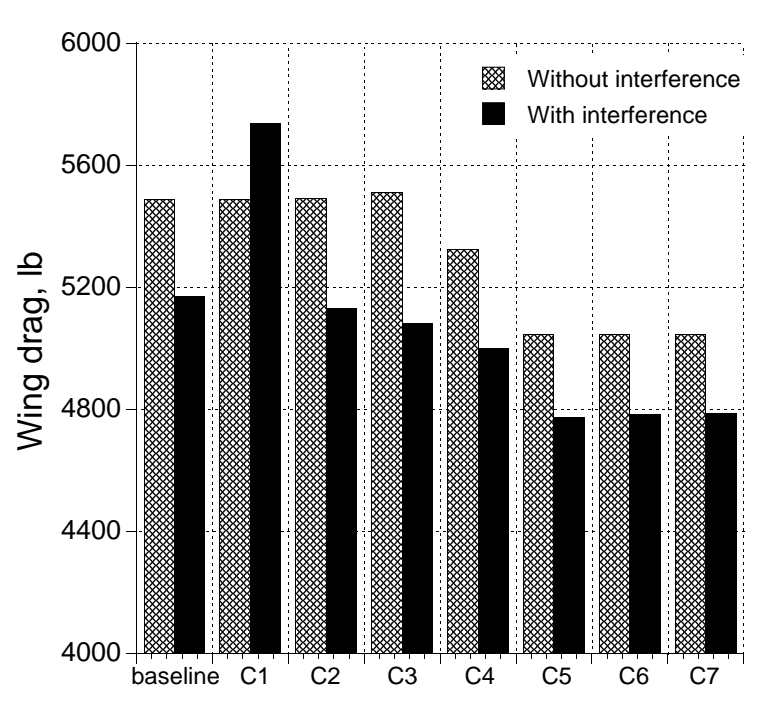

(a) Flapping trim

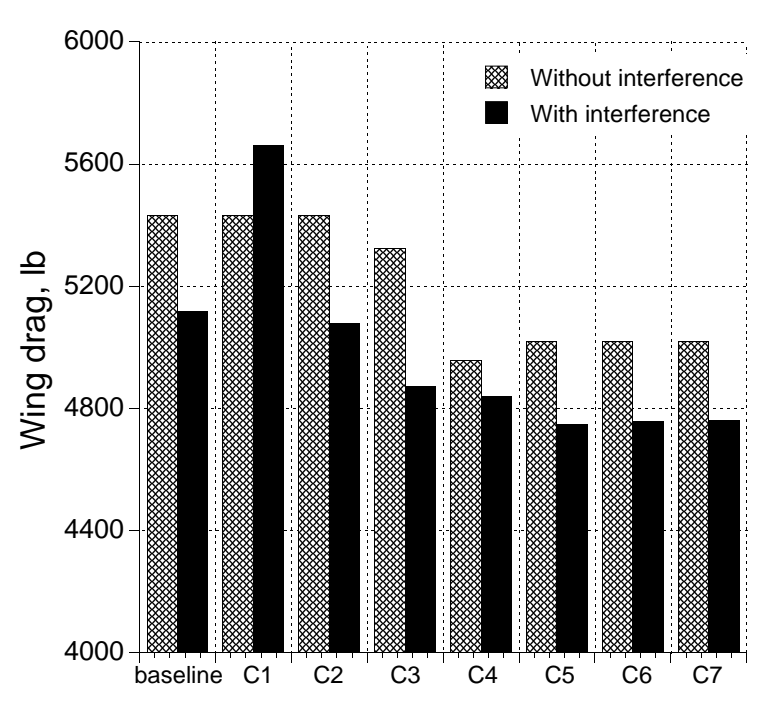

(b) Without flapping trim

Fig. 11: Wing drag of conventional tiltrotor

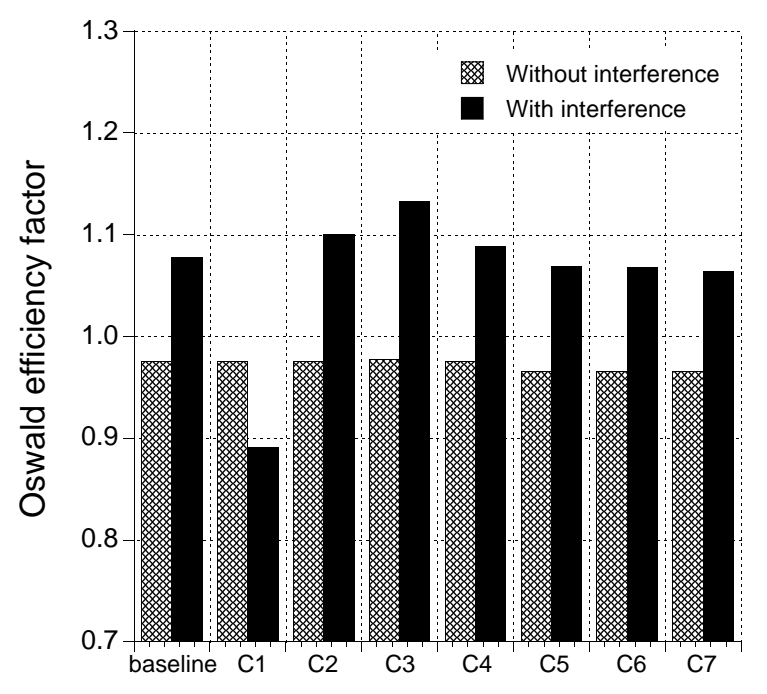

(a) Flapping trim

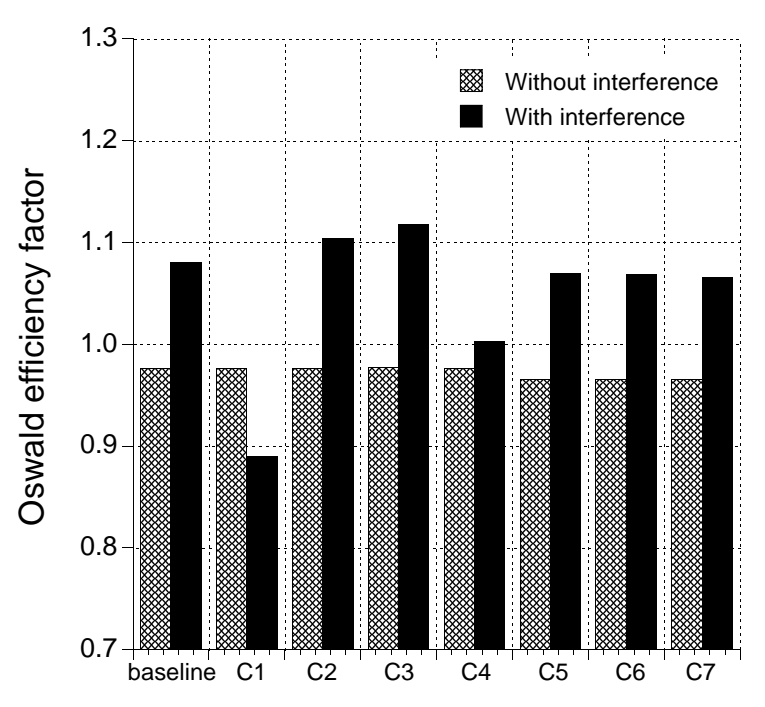

(b) Without flapping trim

Fig. 12: Oswald efficiency factor of conventional tiltrotor 


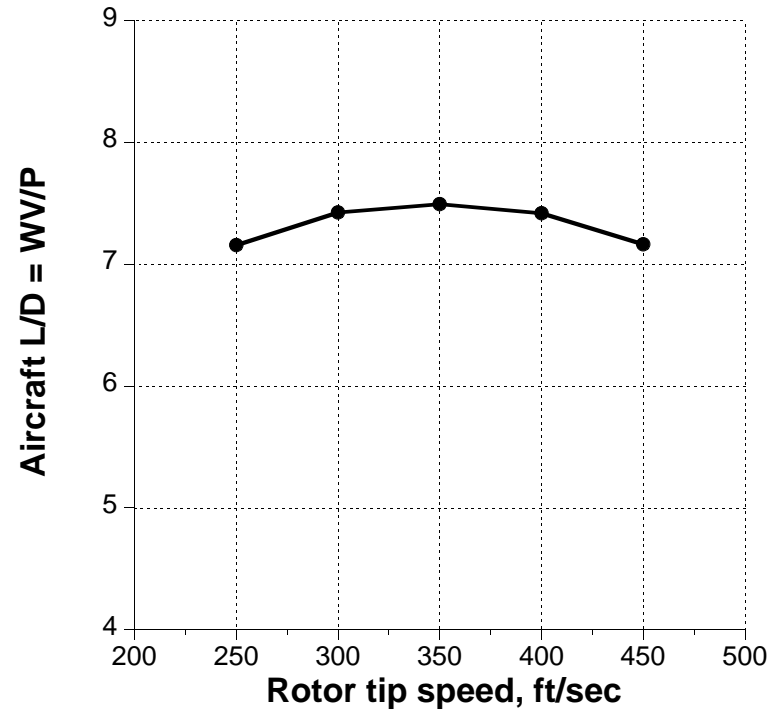

Fig. 13: Aircraft lift-to-drag ratio with rotor tip speed
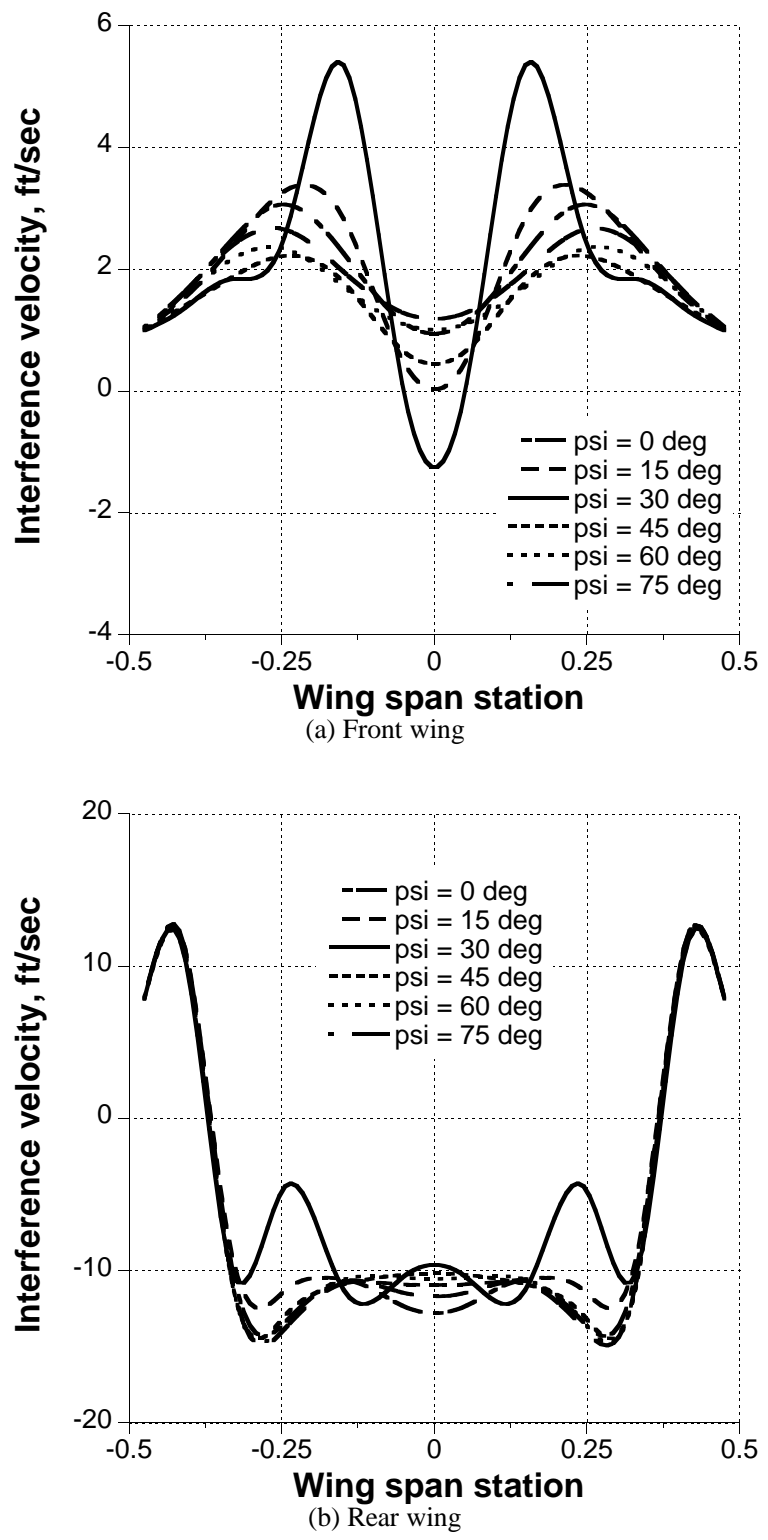

Fig. 14: Interference velocity on the wing of quad tiltrotor (positive upward) 


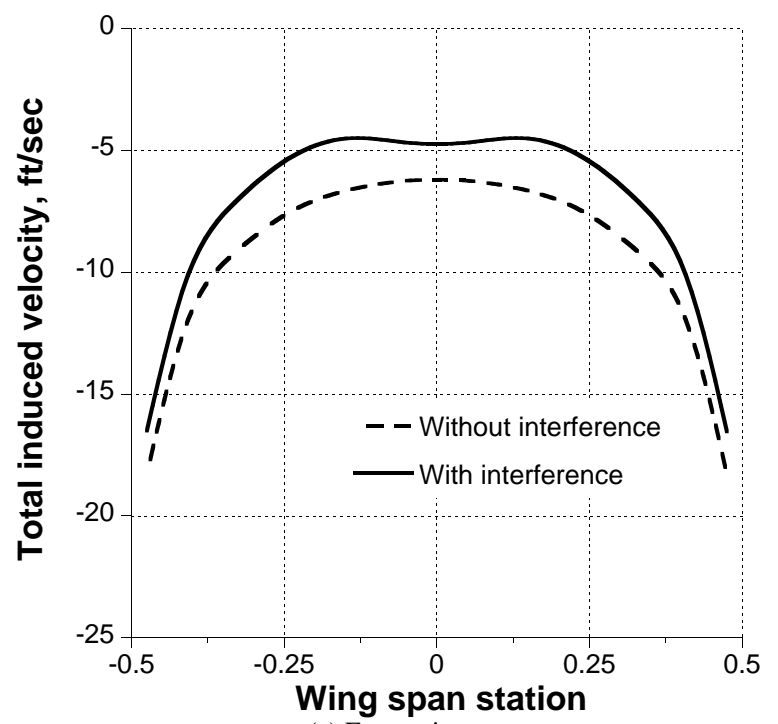

(a) Front wing

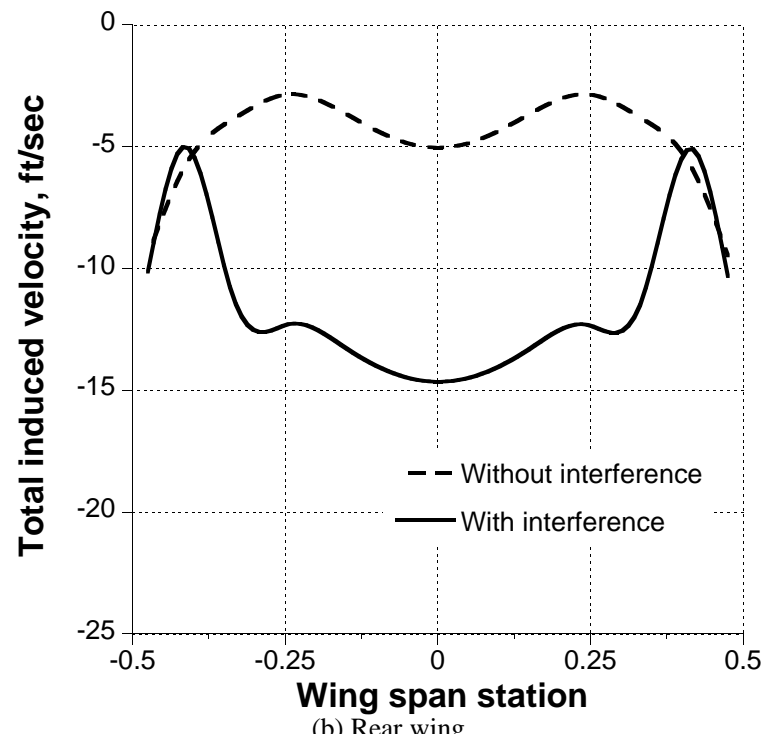

Fig. 15: Total induced velocity on the wing of quad tiltrotor (positive upward)

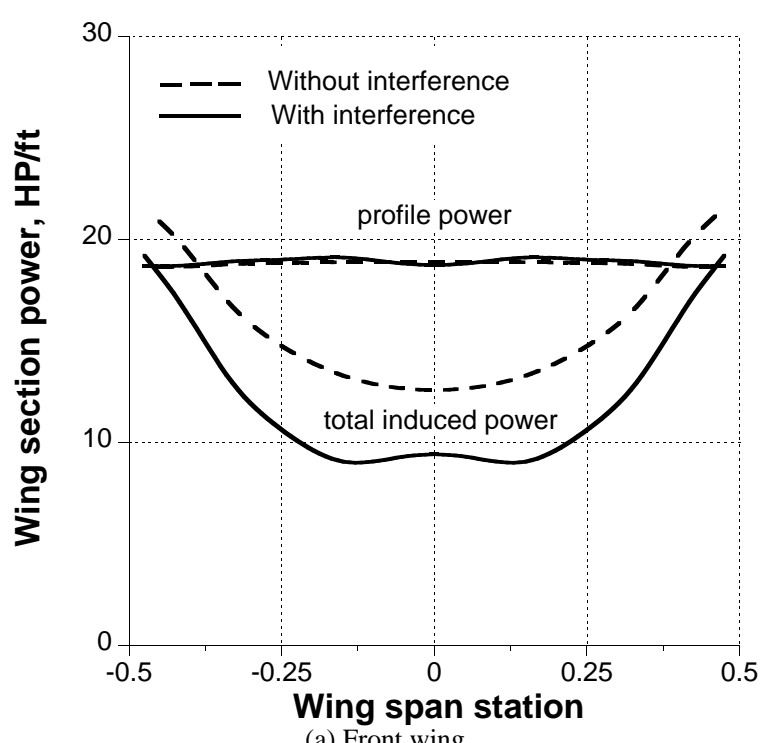

(a) Front wing

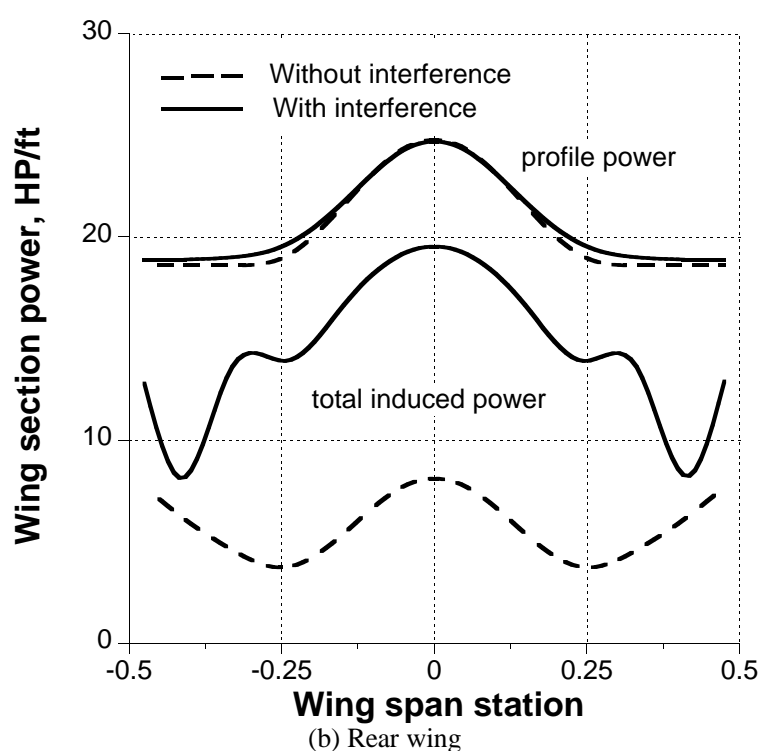

Fig. 16: Wing section power of quad tiltrotor 


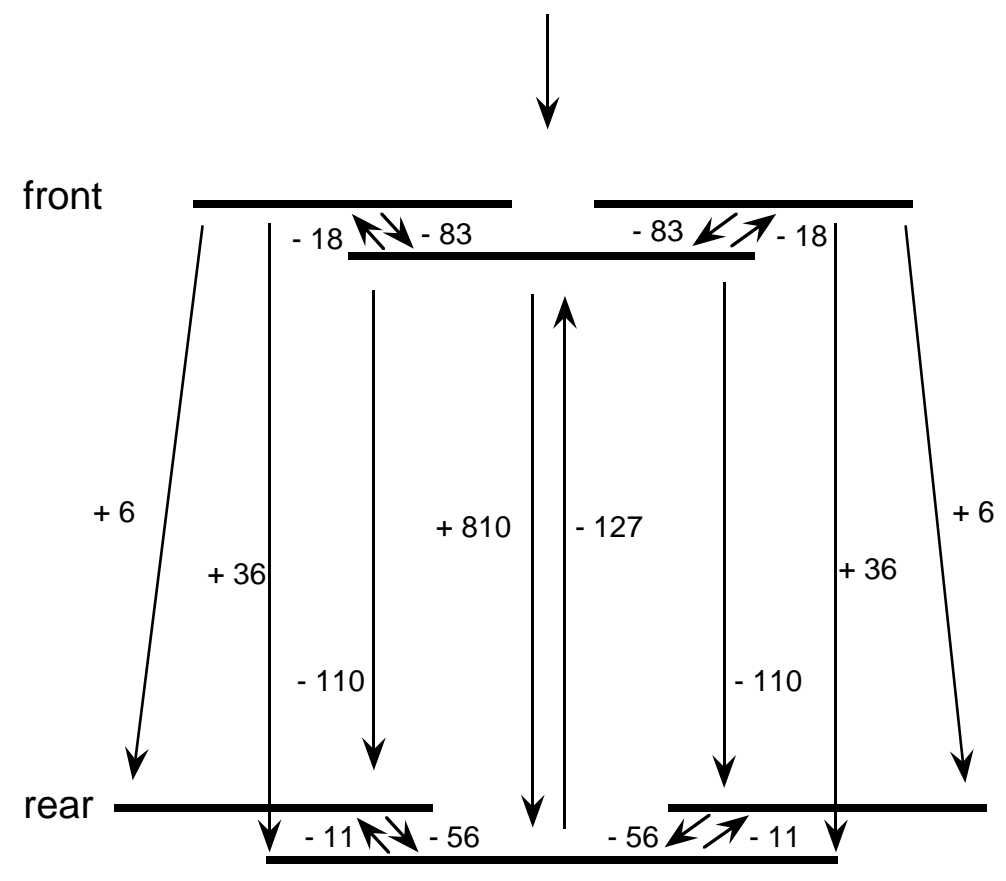

Fig. 17: Interference effect on required power of quad tiltrotor - dimensions are in HP

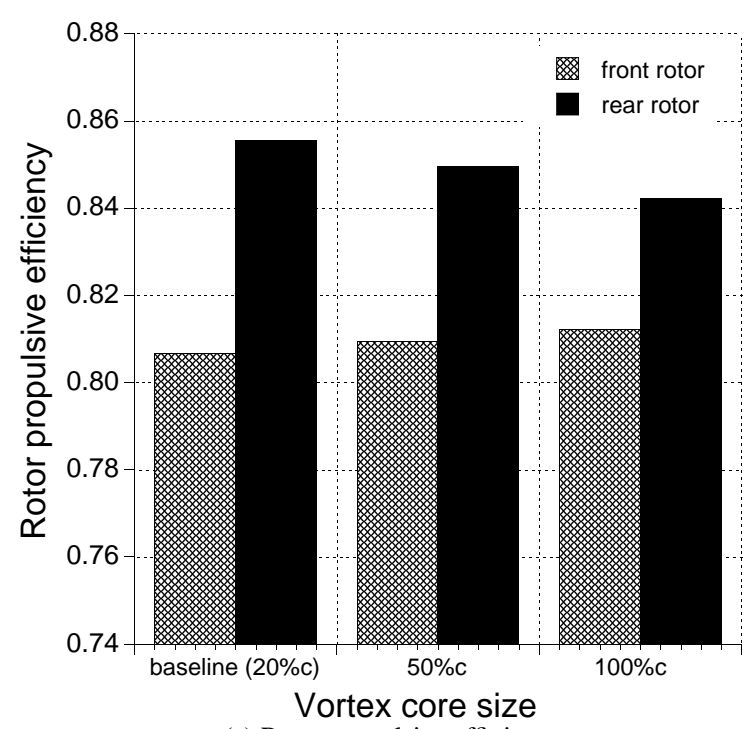

(a) Rotor propulsive efficiency

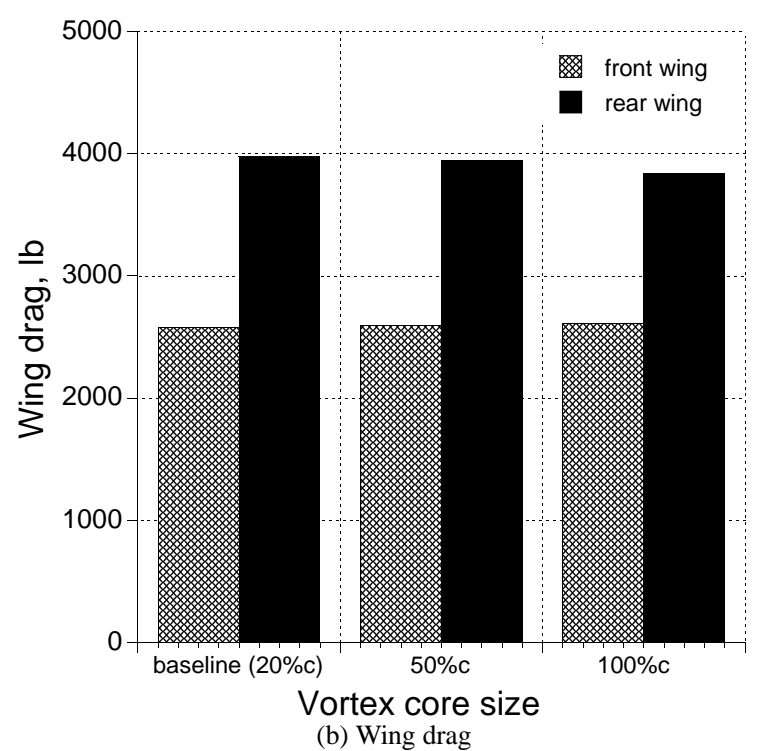

(b) Wing drag

Fig. 18: Effects of vortex core size on aircraft performance 
Baseline

front

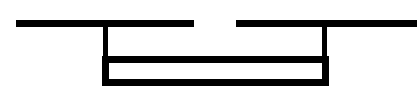

rear

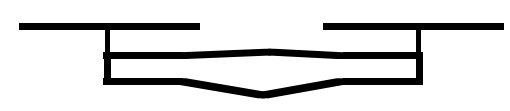

Case 4 (C4)

rear

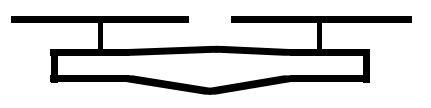

Case 5 (C5)

rear

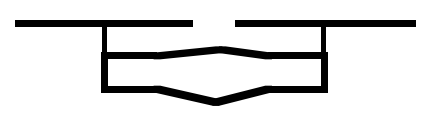

Case $6(\mathrm{C} 6)$

rear

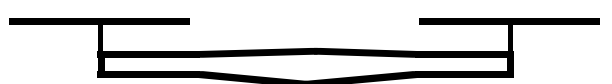

Fig. 19: Rear rotor and wing geometry variations for quad tiltrotor

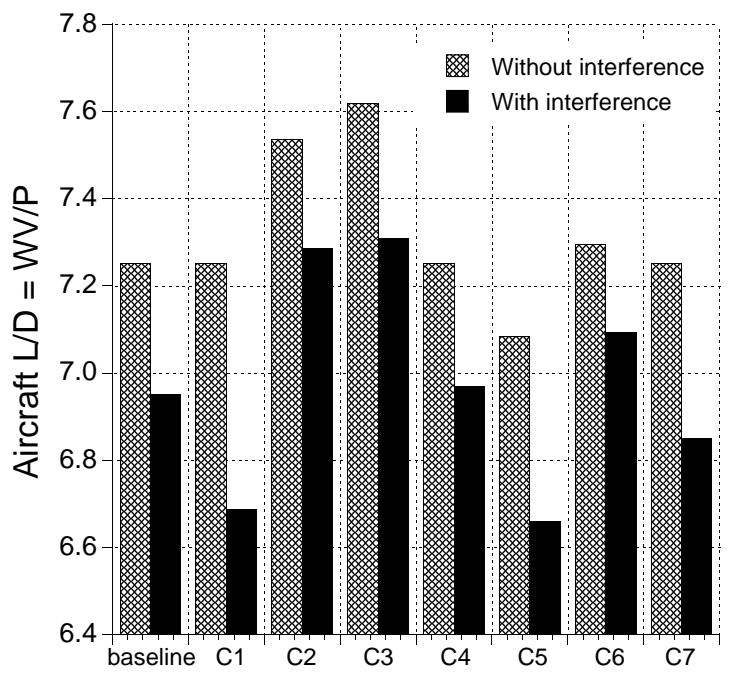

Fig. 20: Aircraft lift-to-drag ratio of quad tiltrotor

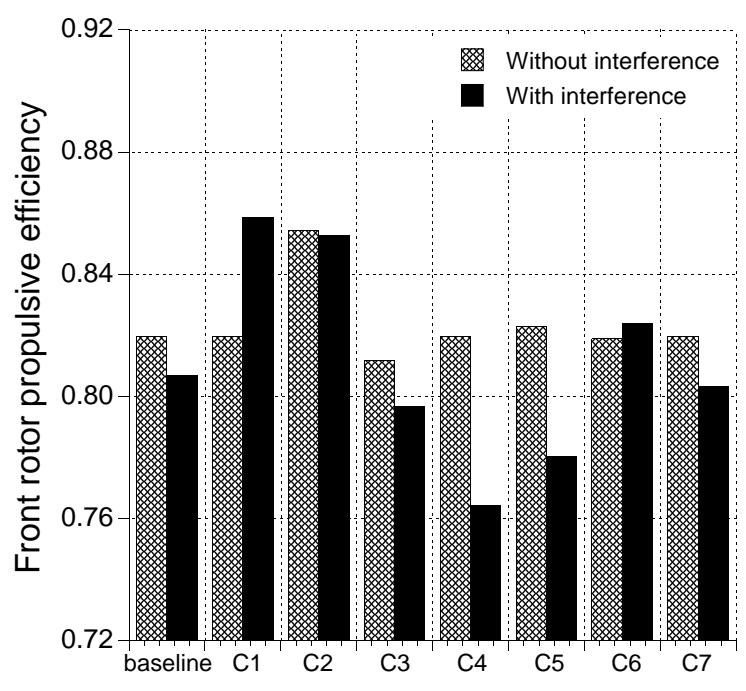

(a) Front rotor

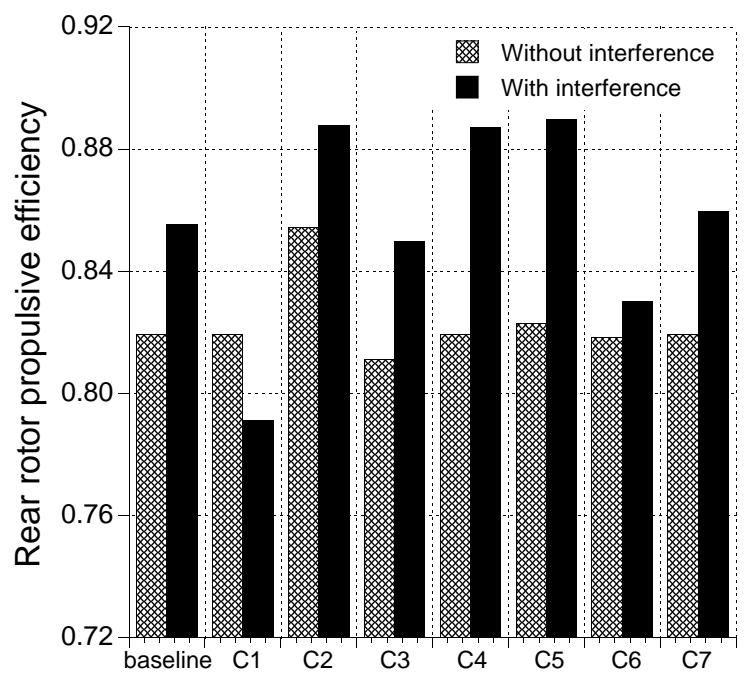

(b) Rear rotor

Fig. 21: Rotor propulsive efficiency of quad tiltrotor 


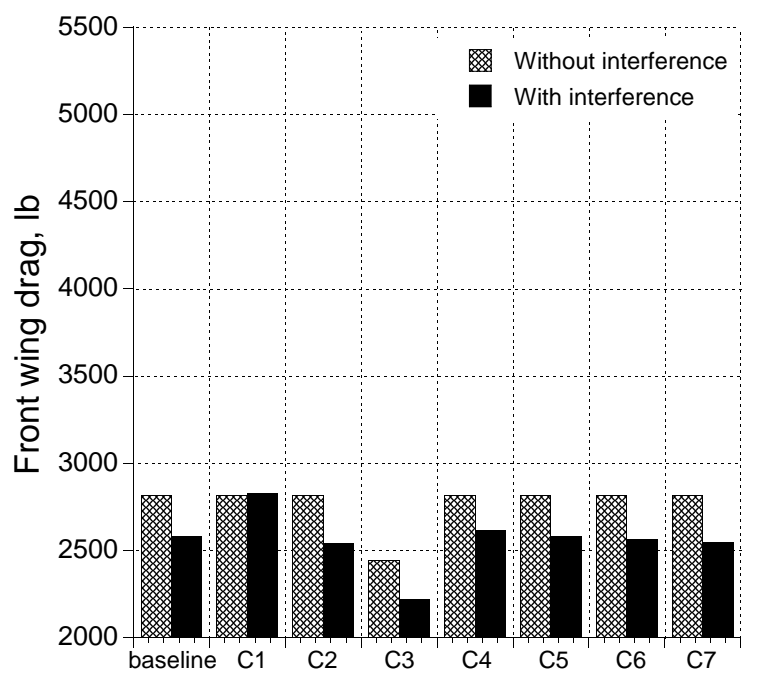

(a) Front wing

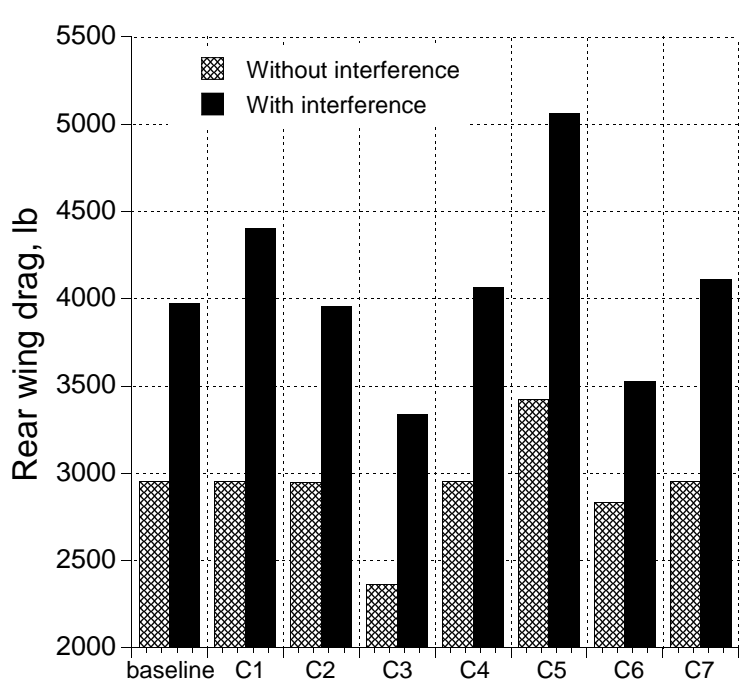

(b) Rear wing

Fig. 22: Wing drag of quad tiltrotor

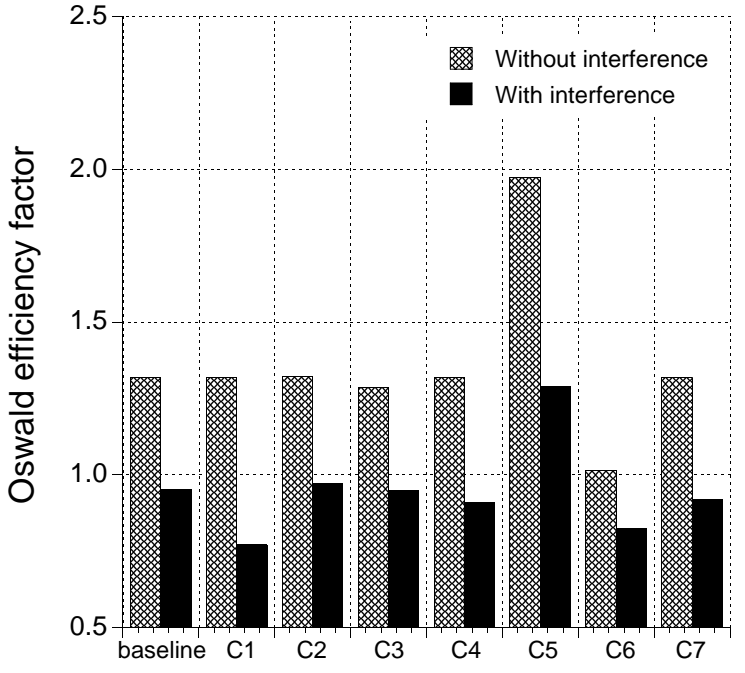

Fig. 23: Oswald efficiency factor of quad tiltrotor 